\title{
Archéopages
}

Archéopages

Archéologie et société

\section{L'élevage du cheval en Languedoc. Le rôle des exploitations agricoles protohistoriques}

Pierre Séjalon, Antoine Ratsimba et Vianney Forest

\section{(2) OpenEdition}

1 Journals

Édition électronique

URL : https://journals.openedition.org/archeopages/285

DOI : 10.4000/archeopages.285

ISSN : 2269-9872

Éditeur

INRAP - Institut national de recherches archéologiques préventives

Édition imprimée

Date de publication : 1 mai 2013

Pagination : 18-25

ISSN : 1622-8545

\section{Référence électronique}

Pierre Séjalon, Antoine Ratsimba et Vianney Forest, « L'élevage du cheval en Languedoc. Le rôle des exploitations agricoles protohistoriques », Archéopages [En ligne], 35 | 10/2012, mis en ligne le 01 octobre 2014, consulté le 26 janvier 2022. URL : http://journals.openedition.org/archeopages/285 DOI : https://doi.org/10.4000/archeopages.285 


\section{L'élevage du cheval en Languedoc Le rôle des exploitations agricoles protohistoriques}

Pierre Séjalon Inrap, UMR 5140 «Archéologie des Sociétés Médititrranéennes »

Antoine Ratsimba Inrap

Vianney Forest Inrap, UMR 5608 «Travaux et Recherches Archéologiques sur les Cultures, les Espacese tles Societétes"

Les analyses archéozoologiques des ensembles provenant de la fouille de deux exploitations agricoles protohistoriques ont conduit à nous interroger d'une part, sur la place du cheval dans l'alimentation carnée des occupants et, d'autre part, sur les lieux délevage du cheval, en comparaison avec les données publiées sur des sites contemporains. En effet, le nombre de restes d'équidés mis au jour sur ces sites était, proportionnellement à la triade domestique (ovicaprins, porcins et bovins), très nettement supérieur à la tendance générale qui se dégageait des études réalisées sur plusieurs habitats groupés languedociens. Le titre de ce numéro d'Archéopages nous a incités à privilégier ici la seconde question.

Nous avons choisi tout d'abord de présenter rapidement les deux sites concernés : Mas de Vignoles IX (Séjalon et al., 2012) et Mas Vigier (Ratsimba et al., 2011) [ill. 1], situés dans le Gard. Après une description sommaire des arguments chronologiques, nous avons mis l'accent sur les vestiges et les résultats susceptibles d'alimenter nos réflexions sur la place des animaux au sein de ces exploitations agricoles. Ensuite, nous proposons une mise en perspective de nos résultats en privilégiant la tranche chronologique comprise entre la fin du VI ${ }^{e}$ et le $\mathrm{IV}^{\mathrm{e}}$ siècle avant notre ère. Cette phase chronologique est considérée en Languedoc comme une période charnière qui voit la création de nombreux habitats groupés, «la civilisation des oppida » chère à Jean Jannoray (Jannoray, 1955). À Nîmes (Gard), en particulier, la population quitte les secteurs de plaine et investit la colline du Mont Cavalier plus au nord pour fonder un oppidum autour de la source de la Fontaine. C'est à partir de cette période qu'apparaît en plaine un important réseau d'exploitations agricoles organisées le long des axes de circulation (Séjalon et al., 2009). Si la plupart ont été détectées au gré des diagnostics sur une fenêtre d'étude de 450 hectares diagnostiqués, environ $10 \%$ ont été fouillées et les données étudiées dans le cadre du Projet Collectif de Recherche «Espace rural et occupation du sol de la région nîmoise, de la Préhistoire à l'époque moderne » .

\section{L'ensemble d'enclos de Mas de Vignoles IX}

L'occupation protohistorique étudiée lors de la fouille de ce site s'organise sous la forme de parcelles limitées par des fossés et localisées de part et d'autre d'un chemin creux [ill. 2a]. La chronologie du site et de son évolution est établie à partir d'un corpus de mobilier céramique mis au jour dans les structures en creux (fossés, fosses et puits). En l'absence de niveaux de sols et de recoupements entre les structures, celle-ci a été limitée à deux phases: la première datée entre 525 et 450 avant notre ère et la seconde entre 450 et $300^{2}$.

Le premier établissement est constitué d'un ensemble de fossés perpendiculaires à la voie qui délimite un espace d'environ $15000 \mathrm{~m}^{2}$ (115 X135 m). Côté est, une rampe a été aménagée pour accéder à l'entrée des parcelles en surplomb par rapport aux niveaux de circulation de la voie. Côté ouest, aucune limite n'a été perçue et la parcelle semble ouverte vers les espaces agraires. C'est dans ce secteur d'interface que sont localisés la plupart des puits qui ont pu être utilisés pour les usages domestiques, pour l'irrigation ou pour abreuver les bêtes. Au sein de cet espace, plusieurs linéaments signalent des découpages internes sans qu'il soit possible d'en définir les fonctions. La localisation des dépotoirs domestiques et la présence de trous de poteaux suggèrent que l'habitat se situe dans l'angle nord-est de la parcelle.

Dans la seconde phase, l'emprise de l'occupation double et un réseau de fossés se développe à l'est 


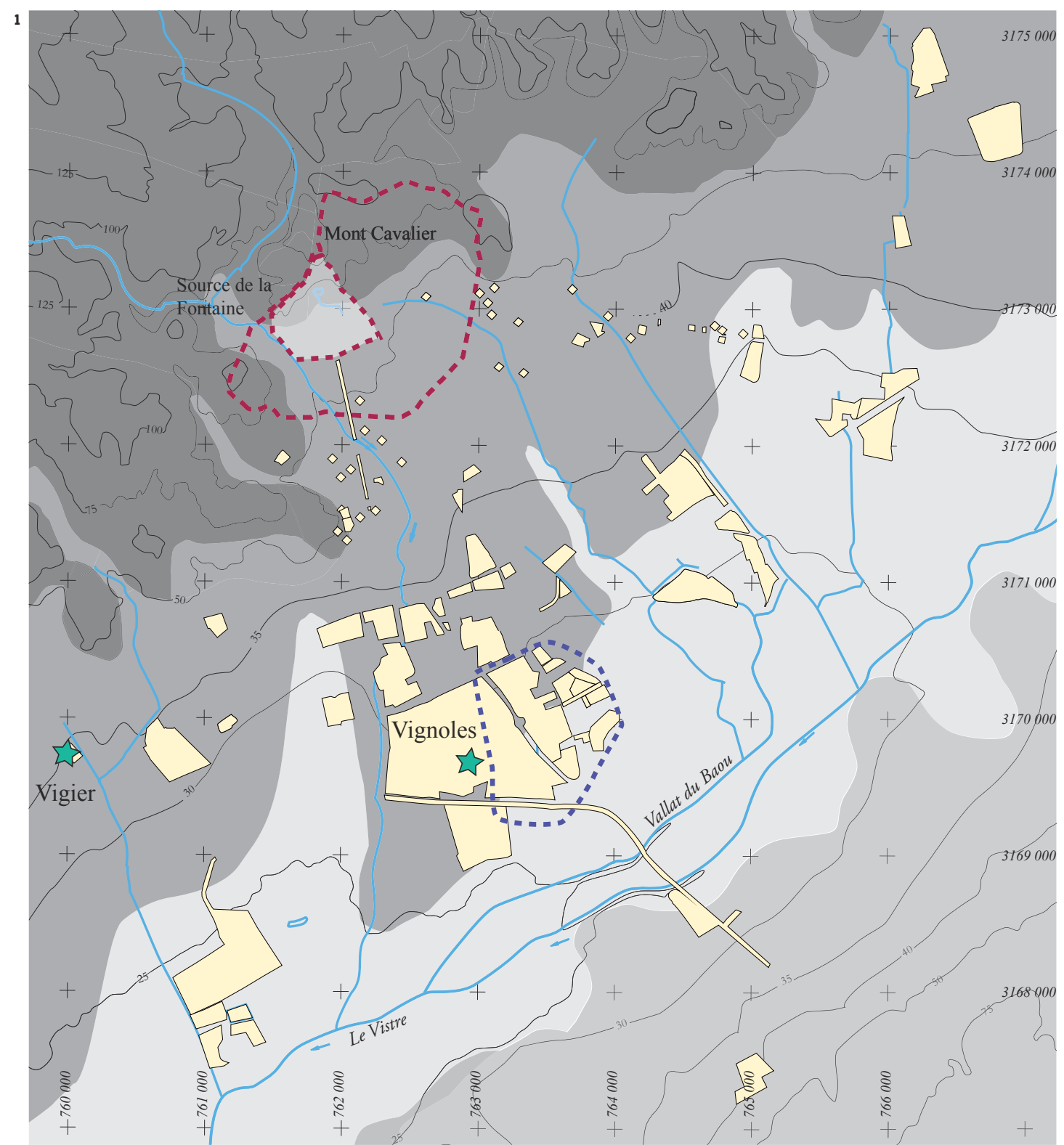

1. Carte des opérations archéologiques réalisées à Nîmes.

[III- Tracé des enceintes protohistoriques et romaines de la ville de Nîmes

\#.... Emprise restituée de l'habitat VIII ${ }^{\mathrm{e}}-\mathrm{VII}^{\mathrm{e}} \mathrm{s}$. av. notre ère

$\curvearrowright$ Localisation des fouilles de Mas de Vignoles IX et de Mas Vigier

Emprise des fouilles et des diagnostics 


\section{Mas de Vignoles IX}

Phase 1 (525/450 av. notre ère)

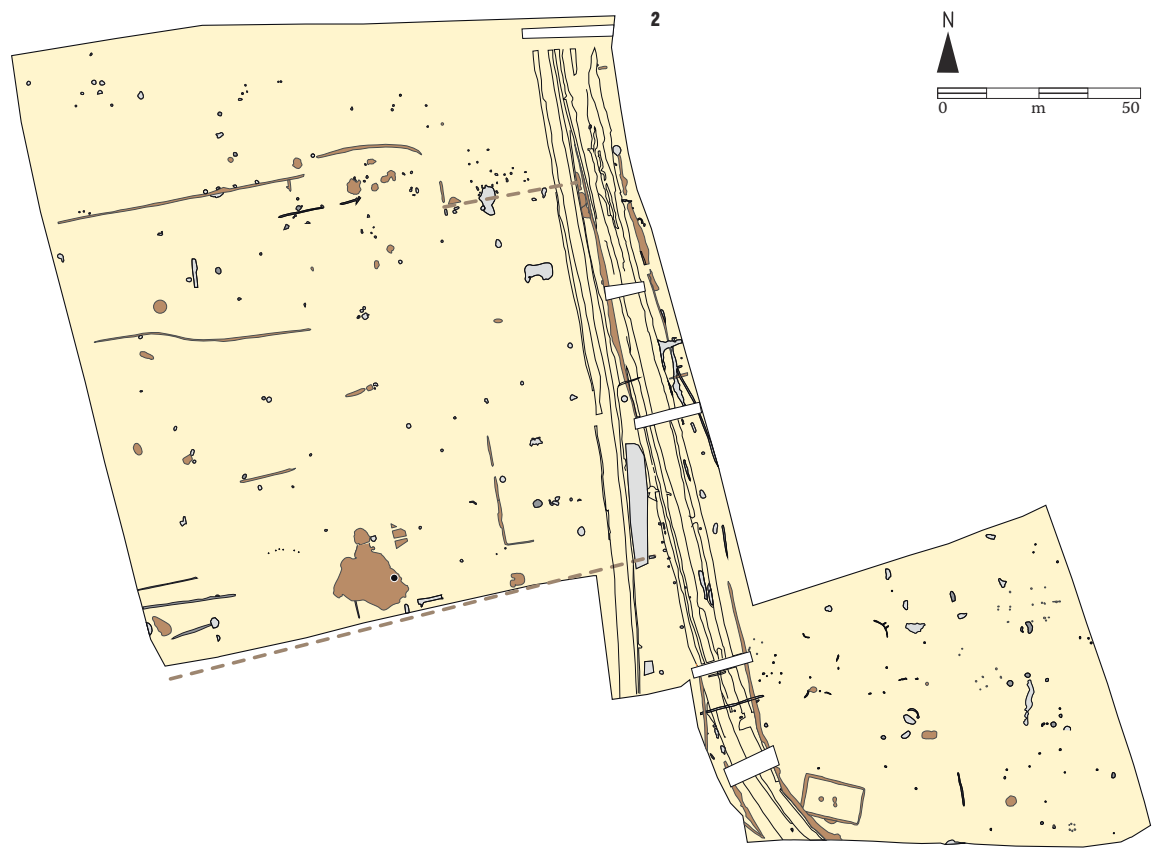

Phase 2 (450/300 av. notre ère)

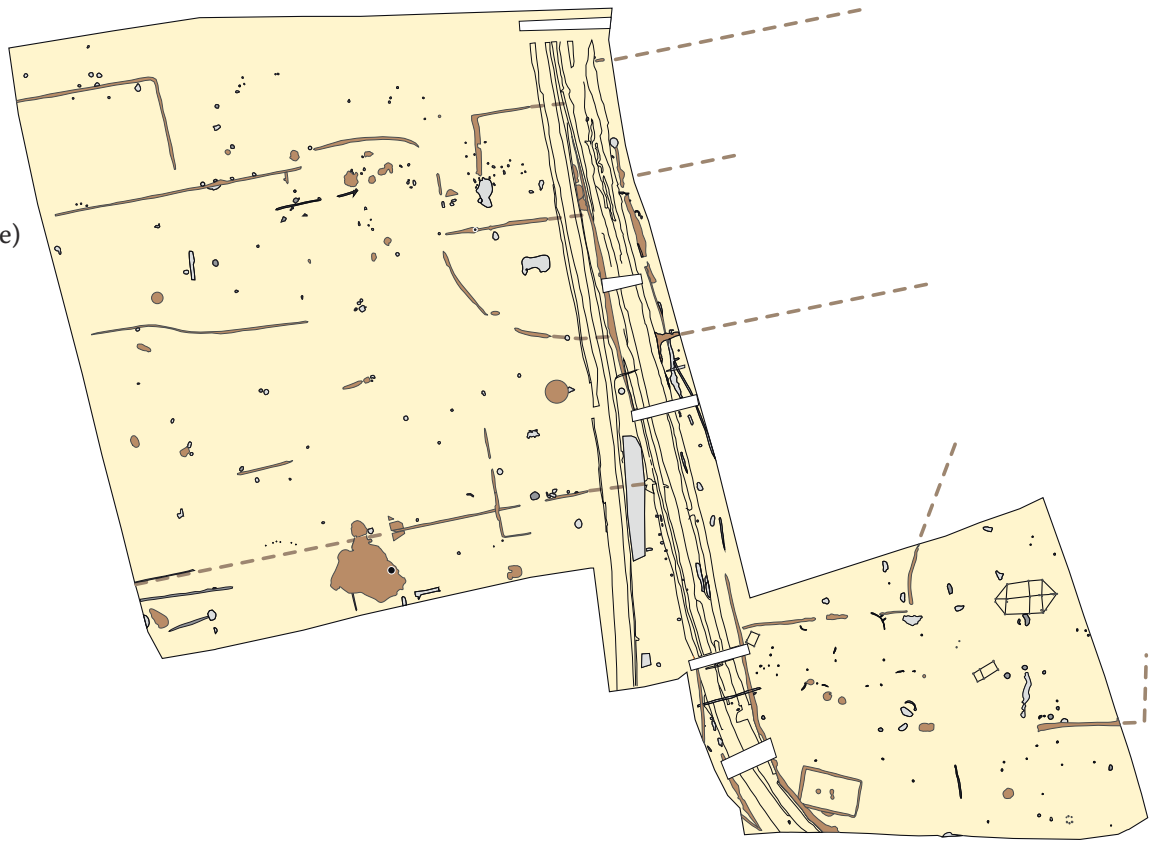

\section{Mas Vigier}

Phase 1 (450/425 av. notre ère)

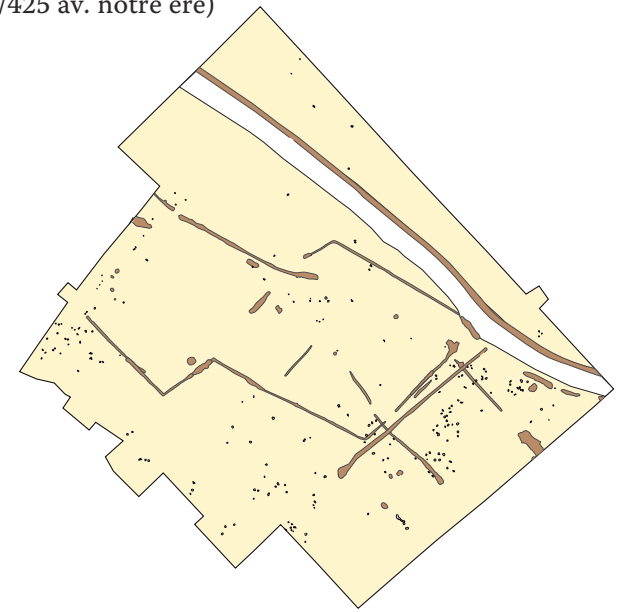

Phase 2 (425/375 av. notre ère)

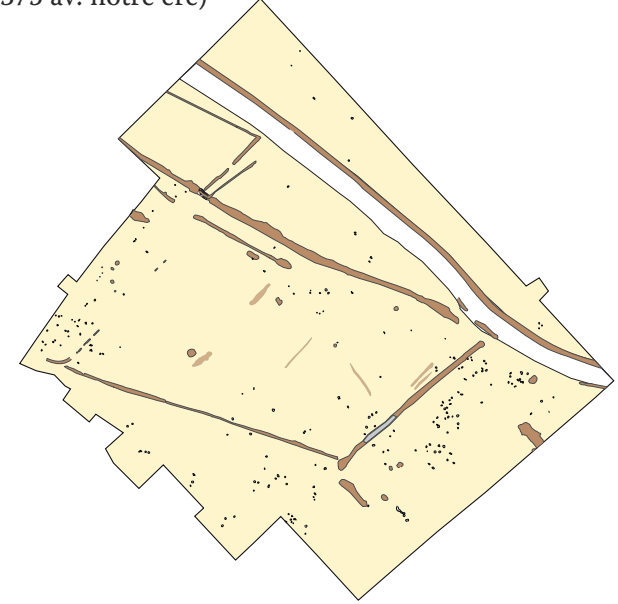


de la voie ${ }^{\mathbf{3}}$. Ils dessinent un parcellaire étalé le long de la voie qui couvre une surface de 18 ooo $\mathrm{m}^{2}$ (200 x 90 m). Côté ouest de la voie, les accès sont réaménagés et deux petits enclos viennent se greffer aux fossés antérieurs : le premier se situe dans l'angle nord-ouest du décapage et mesure au minimum $1000 \mathrm{~m}^{2}$; le second, dans l'angle nord-est de la fenêtre principale et contre la voie côté ouest, mesure $540 \mathrm{~m}^{2}$. Tous deux sont munis d'accès. La fonction de ces petits enclos n'a pas été définie, mais il est possible d'envisager qu'ils aient pu servir au parcage des animaux. Il semble que les bâtiments sur poteaux dégagés au sud-est de l'emprise appartiennent à cette phase. Il s'agit probablement d'une maison à double abside et de deux greniers.

À partir des études menées sur la nature des structures mises au jour, les mobiliers céramiques et les restes végétaux fossiles découverts dans des puits, il est possible d'affirmer que le site de Mas de Vignoles IX correspond à une exploitation agricole qui a évolué dans le temps. Ses occupants, probablement une population réduite, ont habité sur place, générant plusieurs dépotoirs domestiques découverts dans divers comblements de structures en creux. Les 42 ostéorestes déterminés (Forest, 2012) sont issus de 13 de ces structures. Pour la première phase, un fossé, deux puits et une fosse, ont livré 30 restes dont un d'équidé dans un puits. Dans la seconde phase, une fosse sur deux et un fossé sur trois fournissent chacun un reste d'équidé pour un total de sept ostéorestes. Enfin, parmi les cinq ostéorestes issus d'un fossé et de deux fosses, non précisément datés, deux restes d'équidés proviennent d'une des fosses. Les attaques sédimentaires post-dépositionnelles sont nettes et empêchent ainsi l'observation des surfaces, en particulier de marques d'outils. Les restes correspondent à quatre équidés adultes au moins, dont un d'environ six ans. Deux indices osseux sont caballins.

\section{Les enclos de Mas Vigier}

La fouille de ce site, sur 1,5 ha, a mis au jour un vaste réseau essentiellement constitué de palissades et de rares fossés drainants délimitant plusieurs enclos [ill. 2b]. Ces derniers se développent en bordure d'un chemin creux selon un axe sud-est/nord-ouest, sur une longueur observée d'environ $100 \mathrm{~m}$ et sur une largeur de 50 à $70 \mathrm{~m}$. Les déplacements au sein de ces espaces se font grâce à la création de points de passage et d'aires de circulations. Les aménagements les plus anciens remontent aux alentours de 450 avant notre ère. Le site connaît une durée d'occupation relativement courte puisqu'il semble abandonné dès 375 avant notre ère. Cette chronologie est fondée sur l'étude d'un lot de céramiques ${ }^{4}$.

La bonne conservation des structures et leur fouille méthodique, quasi exhaustive, a permis non seulement de percevoir les évolutions successives de cette occupation rurale, mais aussi de déterminer la nature de la plupart des aménagements. Ainsi, plusieurs segments fossoyés correspondent-ils à des tranchées d'installation de palissades. La présence de poteaux ou de piquets a pu y être clairement mise en évidence. Leurs diamètres varient de 10 à $40 \mathrm{~cm}$. Ils sont soit coalescents, soit espacés de 10 à $20 \mathrm{~cm}$. Dès 450 (phase 1), une vaste parcelle formée de deux espaces de respectivement 1 ooo et $1600 \mathrm{~m}^{2}$ est clôturée, bordée au sud par une aire de $400 \mathrm{~m}^{2}$ partiellement enclose.

Au cours du quart de siècle suivant (phase 2), le parcellaire est totalement réaménagé. Cette nouvelle organisation, qui s'appuie en partie sur les orientations antérieures, est marquée par la réunion et l'agrandissement des deux enclos précédents. Le nouvel espace ainsi créé atteint une surface de $4.500 \mathrm{~m}^{2}$; il n'est plus totalement cerné de clôtures, mais délimité à l'est par un fossé drainant. Un nouveau parc clôturé est situé au nord de l'emprise et observé sur $400 \mathrm{~m}^{2}$, dans l'espace entre l'enclos et la voie. Ensuite, le parcellaire ne connaît plus que des transformations minimes (phase 3), en relation avec l'entretien des aménagements drainants ou avec des modifications partielles des fossés préexistants.

Les fonctions des espaces sont déduites de la nature du mobilier céramique, de fragments d'une plaque-foyer décorée, de chenets, d'adobe fondu et de blocs équarris, récoltés dans les comblements de fosses et de fossés. Différents indices laissent penser que des bâtiments s'élevaient sur les bordures sud-est et nord-ouest du parcellaire. En effet, au nord-ouest, des trous de poteaux indéniablement protohistoriques ont été mis au jour, alors qu'au sud-est, les restes de la destruction d'un bâtiment ont été découverts dans un fossé. De plus, les axes de cheminement vers la voie mènent à ces deux secteurs et ces espaces ne sont pas entièrement délimités contrairement aux enclos centraux. La fonction de ces derniers peut être avancée à partir des résultats des analyses malacologiques (Martin, 2011). Plusieurs couches de comblement de ces fossés ont fait l'objet de prélèvements. Ils ont permis la mise en évidence d'assemblages typiques d'escargots (Candidula gigaxii et Candidula unifasciata/rugosiuscula) qui sont symptomatiques des pelouses sur-pâturées par les ovins (Labaune, Magnin, 2002) et qui signalent ici que les espaces environnants étaient marqués par un pastoralisme intensif. Cela suggère que ces enclos pouvaient être destinés au parcage de bétail.

Les 102 ostéorestes déterminés (Forest, 2011) proviennent de 20 faits qui se répartissent en quatre lots chronologiques correspondant aux trois phases d'organisation des structures. Pour la phase 1 , sept fossés et une fosse livrent un total de 22 restes dont un d'équidé. La phase 2, constituée de sept fossés, apporte 38 restes dont 11 d'équidés dans quatre structures. Enfin la phase 3, considérée comme la période d'abandon, est documentée par un fossé qui a fourni deux restes d'équidés sur un total de 19. Un quatrième groupe, trois fosses et 


\begin{tabular}{|c|c|c|c|c|c|c|c|c|c|}
\hline site & phase & Ovicaprins & Porcins & Bovins & Équidés & Cerf & Chien & total NR & indéterminés \\
\hline \multirow[t]{4}{*}{ Mas de Vignoles IX } & 1 & 20 & & 9 & 1 & & & 30 & 7 \\
\hline & 2 & 1 & 1 & 2 & 2 & 1 & & 7 & 10 \\
\hline & 1 ou 2 & 1 & & 1 & 2 & & 1 & 5 & 2 \\
\hline & total NR & 22 & 1 & 12 & 5 & 1 & 1 & 42 & 19 \\
\hline \multirow[t]{5}{*}{ Mas de Vigier } & 1 & 6 & & 15 & 1 & & & 22 & 25 \\
\hline & 2 & 2 & & 20 & 11 & 3 & 2 & 38 & 26 \\
\hline & 3 & 5 & 4 & 8 & 2 & & & 19 & 27 \\
\hline & 1 à 3 & 12 & 3 & 6 & 1 & & 1 & 23 & 94 \\
\hline & total NR & 25 & 7 & 49 & 15 & 3 & 3 & 102 & 172 \\
\hline
\end{tabular}

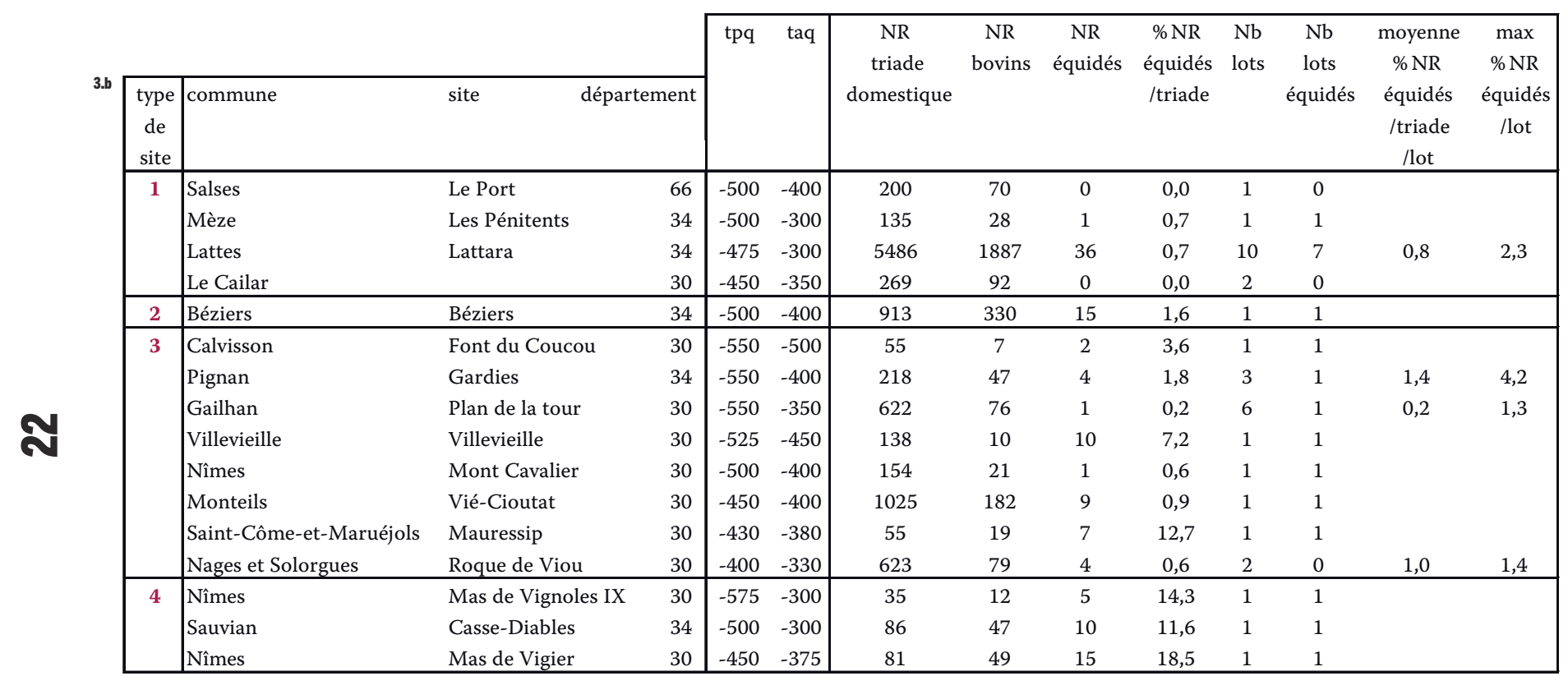

3. a. Nombres de restes (NR) par taxon et par phase dans les deux sites nîmois présentés;

b. Récapitulatif des résultats archéozoologiques sur les sites d'habitat du Languedoc méditerranéen du milieu du vie $s$. à la fin du Ive $S$. avant notre ère.
5. Sites fouillés entre 1991 et 2012 ; études archéozoologiques menées par Colomer Arcas; Columeau,

CNRS, UMR 6573,

Centre Camille Jullian;

Forest, Inrap;

Gardeisen, CNRS,

UMR 5140 «Archéologie

des Sociétés

méditerranéennes».

6. Afin de ne pas occulter

de données et de respecter

l'espace et la lisibilité

de la publication. un fossé, n'a pu être attribué à l'une des trois phases : il contient un reste d'équidé sur 23 ostéorestes. Les ossements ont été violemment altérés par les sédiments, action qui rend incertaine l'identification de quatre fragments d'équidés et impossible l'observation de traces d'outils. Il s'agit de trois ou quatre équidés adultes, dont un jeune, un autre plutôt âgé, et un mâle. Quatre indices sont caballins et une dent est de morphologie asinienne. La plus grande partie de la variabilité ostéométrique des équidés gaulois (Forest, 2008) est couverte par l'échantillon.

\section{Mise en perspective}

L'abondance relative des restes d'équidés dans les séries mises au jour à Mas de Vignoles IX et à Mas Vigier [ill. 3a], par rapport aux autres sites contemporains, en particulier les oppida proches, nous a conduit à évaluer la présence de ces restes dans une approche synthétique régionale ${ }^{\mathbf{5}}$. Nous nous sommes limités au Languedoc méditerranéen pour opérer dans une unité géographique, culturelle et archéozoologique homogène [ill. 3b]. 16 sites seulement ont fait l'objet d'études archéozoologiques entre le milieu du vi ${ }^{\mathrm{e}}$ et la fin $\mathrm{du} \mathrm{IV}^{\mathrm{e}}$ siècle avant notre ère. Elles ont été réalisées principalement par Philippe Columeau (Columeau, 1991, 1997a, 1997b, 2004) et sont en conséquence déjà anciennes. Nous avons suivi le découpage des séries par phases chronologiques successives (générant chacune un lot osseux) au sein de chaque site, comme l'a pratiqué cet auteur, et comme l'a poursuivi globalement Armelle Gardeisen pour les séries de Lattara (Colomer Arcas, Gardeisen, 1992) et du Cailar (Gardeisen, 2002). Comme ce degré de précision n'a pas apporté d'informations autres, nous avons choisi de présenter des résultats globaux par site, tout en livrant quelques éléments de détail ${ }^{\mathbf{6}}$. Nous avons regroupé les sites suivant leur nature : quatre 
agglomérations lagunaires, huit agglomérations perchées (pour ne pas parler d'oppida) plutôt apparentées au type du village, une qui serait plutôt une ville (Béziers, Hérault) et trois sites ruraux (Mas Vigier, Mas de Vignoles et la ferme de Sauvian/Casse-Diables, à proximité de Béziers). Nous n'avons pas retenu le site de Mas de Vignoles IX car le nombre de restes (NR) total de la triade domestique n'atteint pas la valeur seuil de 50 pour prendre en compte un site. Les résultats archéozoologiques de ce site sont similaires à ceux de Mas de Vigier, qui sert de référence pour les deux.

Dans un premier temps, l'importance du NR d'équidés est testée par rapport à celui de la triade domestique qui constitue le socle référentiel des assemblages osseux en Languedoc depuis le Néolithique [ill. 3b et 4a]. La proportion maximale des équidés est atteinte à Mas Vigier avec $16 \%$ et dépasse $10 \%$ à Casse-Diables. À l'opposé, les agglomérations lagunaires ou la ville de Béziers sont pauvres en restes, au maximum 1,6 \% durant une phase de Lattara. La moitié des huit agglomérations perchées de type village rejoignent les précédentes tandis que deux autres approchent ou dépassent $7 \%$ : Mauressip et Villevieille (Gard). Dans la limite du volume de l'échantillon, il ressort que les habitats ruraux livrent davantage de restes d'équidés que les agglomérations. Ce constat est particulièrement net pour des sites voisins, entre Mas Vigier, Mas de Vignoles IX et l'agglomération du Mont Cavalier, et une centaine de kilomètres plus à l'ouest, entre la ferme de Sauvian/CasseDiables et la ville de Béziers. De plus, il se dégage une gradation au sein des quatre catégories de sites suivant leur nature fonctionnelle : plus la catégorie est a priori portée vers l'agriculture et plus la proportion de restes d'équidés est forte. Il est à préciser qu'aucune évolution chronologique ne s'est imposée, ni dans l'échantillon, ni au sein de chaque site ${ }^{7}$.

Avant d'essayer d'interpréter ce résultat en termes d'élevage, il faut définir la nature des vestiges osseux. En l'absence de tout indice pouvant lier cette abondance à une activité singulière, par exemple cultuelle, l'alimentation carnée est la source la plus probable des restes. Sur des sites où les ossements sont en bon état, la présence de traces de découpe irait dans ce sens comme à Lattara (Gardeisen, 1999, 2003, 2010). Sur les sites de Mas Vigier et de Mas de Vignoles, le mélange des ossements d'équidés avec ceux très probablement alimentaires de la triade domestique serait un second argument.

Les bovins étant eux aussi fortement représentés dans les assemblages des trois sites ruraux [ill. 3a et 4b], il est nécessaire au préalable de vérifier que l'abondance relative des équidés n'est pas le fruit d'une déformation de la composition des séries sous l'effet d'une fonte taphonomique postdépositionnelle différentielle qui favoriserait les gros os par rapport à ceux des taxons plus petits que sont les ovicaprins et les porcins. Dans les agglomérations où la conservation de la matière osseuse est bonne et où les restes de porcins et d'ovicaprins sont nombreux (c'est-à-dire où cette fonte est faible), les bovins sont nettement mieux représentés que les équidés. Le net découplage dans les agglomérations entre les restes d'équidés et de bovins, ainsi que l'écart de la part des restes de bovins dans la triade entre les agglomérations perchées de type village et les sites lagunaires ou Béziers, suggèrent que la fonte taphonomique post-dépositionnelle joue un rôle mineur dans l'abondance relative de restes d'équidés [ill. 4b].

Quelle est la cause de cette consommation plus forte des équidés dans les milieux ruraux? Il est certes possible de retenir une attirance de leurs populations pour la viande d'équidés plus prononcée que celle des habitants des agglomérations. Toutefois, cet argument perd de sa force pour expliquer la plus grande consommation de bovins dans les agglomérations lagunaires et à Béziers par rapport aux agglomérations perchées.

La topographie des sites s'impose donc comme facteur explicatif prééminent. Non qu'il soit plus difficile d'amener des bovins vivants dans les agglomérations perchées que dans les sites de plaines, puisque Béziers prouve le contraire. Mais plus simplement, à défaut d'une grande attractivité commerciale qui pourrait caractériser les sites lagunaires et Béziers, les agglomérations perchées et les sites ruraux s'alimentent prioritairement sur les troupeaux qui les environnent... Autrement dit, ils les élèvent, si l'on postule que les habitants de ces types de sites exploitent eux-mêmes le finage qui les entoure, et ils en consomment les produits de manière principalement autarcique, contrairement aux sites lagunaires et à Béziers. Dans cette hypothèse, les sites ruraux tels que Mas de Vignoles, Mas Vigier ou Casse-Diables, seraient les lieux d'un élevage plus orienté vers les équidés et les bovins, statut qu'ils pourraient partager avec certaines agglomérations perchées comme Mauressip et Villevieille.

En conséquence, notre étude semble montrer secondairement un net tropisme de l'élevage bovin pour la plaine languedocienne, donc lié à la topographie, et des modes de consommation qui diffèrent selon la nature des sites. Nos conclusions sont contraires à celles d'Armelle Gardeisen dans sa synthèse sur la «Gestion des animaux de bouche au cours du $\mathrm{V}^{\mathrm{e}}$ siècle avant notre ère» (Gardeisen, 2010), preuve de la complexité de l'interprétation des résultats archéozoologiques en termes de pratiques humaines dont le thème ultime, dans le cheminement logique à partir des ossements, est l'élevage. Les équidés que les habitants produisaient auraient été vendus comme animaux de monte ou de bât dans les autres sites où, en conséquence, ils n'avaient pas vocation à assurer une consommation régulière comme les bovins sur les sites ruraux. Les animaux en fin de vie, à mort précoce par accident (malformation, inadaptation, pathologie...) ou tardive par réforme, auraient été mangés pour «ne pas gâcher». D'autres indices peuvent être
7. Toutefois, sur le site plus récentes connaissent un léger décollement de 1 à $2 \%$ qui se poursut durant le $\mathrm{III}^{\mathrm{e}} \mathrm{s}$. avan notre ère. 
4.a. Équidés, importance

relative par rapport

à la triade domestique

en fonction du type de site

$(\%=$ NR équidés/NR triade

domestique *100 (phase

maximale).

b.Abondance comparée des

restes d'équidés et de bovins. 4.a

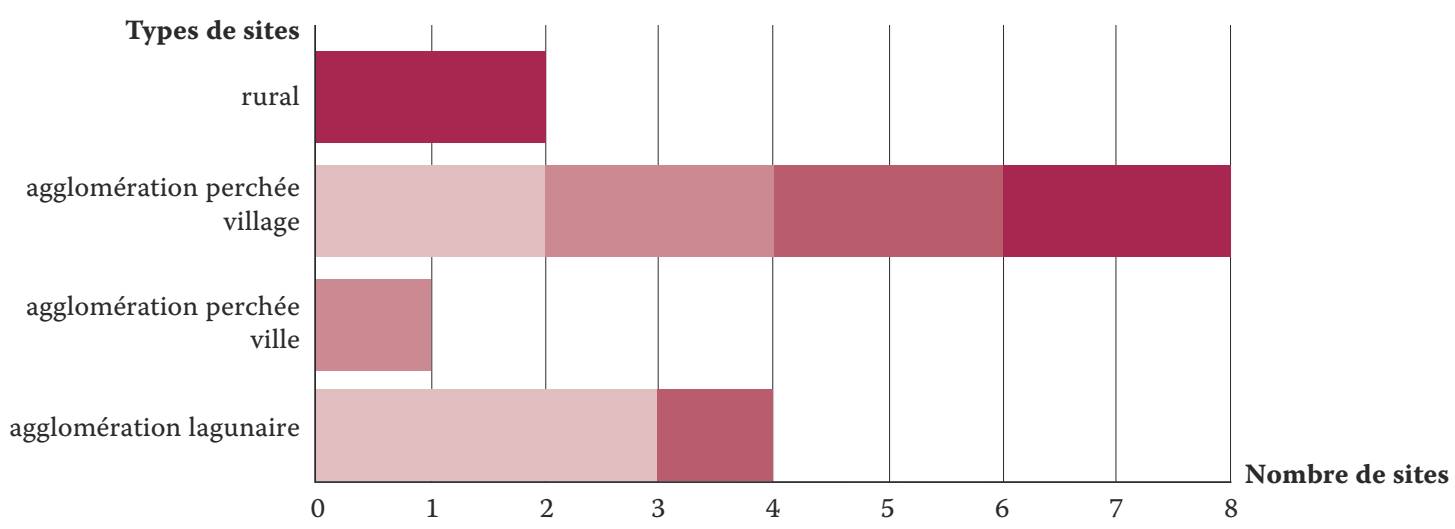

4.b

NR bovins / NR triade domestique "100

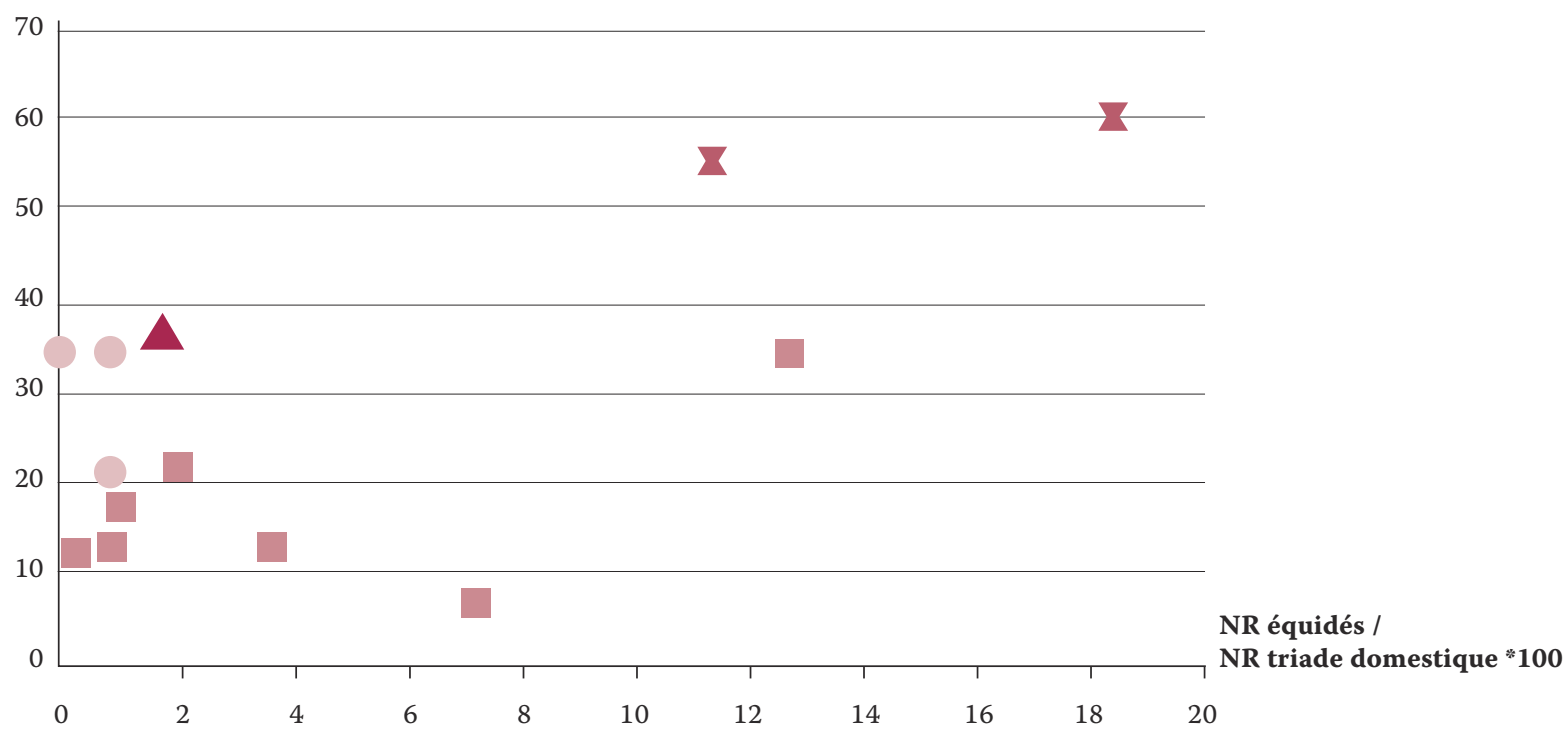


mobilisés pour asseoir l'hypothèse d'un élevage plus développé des équidés sur ces sites ruraux. Malheureusement, la poignée de données d'âge ou de sexe, ne nous permet pas aujourd'hui de nous prononcer. Aucun reste de poulain, par exemple, n'a été retrouvé, tout au plus pouvons-nous affirmer qu'il s'agit majoritairement de chevaux. Lâne et ses hybrides sont signalés, mais leur importance numérique demeure à ce jour très mal documentée.

En l'état d'une documentation réduite pour le $\mathrm{v}^{\mathrm{e}}$ siècle avant notre ère en Languedoc méditerranéen et par rapport à la question posée au départ, cette rapide enquête apporte un faisceau convergent de résultats qui suggèrent que les exploitations agricoles ont joué un rôle non négligeable dans l'élevage du cheval. Du point de vue des structures archéologiques, la caractérisation des fossés par la mise en évidence de palissades et d'accès a permis d'avancer l'hypothèse que certains enclos pouvaient être destinés au parcage du bétail, dont les équidés. Celle-ci s'est vue confirmée au Mas Vigier grâce aux résultats des analyses malacologiques. Du point de vue de l'analyse archéozoologique, il a pu être montré que la part relativement forte des restes d'équidés dans la consommation carnée relevait probablement d'un accès facilité à cette viande parce que ces exploitations agricoles consacraient une part plus importante de leur activité d'élevage au cheval que la plupart des autres sites du Languedoc.

Les situations similaires observées à Nîmes et à Béziers dessinent un trait supplémentaire d'un schéma économique de complémentarité entre les sites producteurs, les exploitations agricoles, et les sites consommateurs, les habitats groupés (Pomarèdes et al., à paraître). Connaissant la place du cheval dans la société gauloise du Midi (Arcelin, 2005), ce premier travail ouvre des perspectives à venir sur le statut des habitants de ces exploitations agricoles, en particulier vis-à-vis des agglomérations voisines et sur les formes de l'élevage de ces animaux.

\section{Références bibliographiques}

ARCELIN P., 2005, «Les équidés dans l'iconographie de la Gaule méditerranéenne à lâge du Fer », in GARDEISEN A. (DIR.), Les équidés dans le monde méditerranéen antique, Actes du colloque d'Athènes, 26-28 novembre 2003, Monographie d'Archéologie Méditerranéenne, Hors-série, 1, Lattes, Édition pour le Développement de l'Archéologie en Languedoc-Roussillon, p. 249-266.

Colomer Arcas A., Gardeisen A., 1992, « La consommation des animaux d'élevage et de chasse dans la ville de Lattara (fin du IV ${ }^{\mathrm{e}} \mathrm{s}$. av. n. è.-milieu du $\mathrm{I}^{\mathrm{er}}$ s. de n. è.) », in Py M. (DIR.), Recherches sur l'économie vivrière des Lattarenses, Lattara, 5 , Lattes, CNRS, p. 91-110.

Columeau P., 1991, L'Animal pour l'homme, recherches sur l'alimentation carnée dans le sud de la France du Néolithique au Moyen Âge d'après les vestiges osseux. I: le monde rural, Travaux du Centre Camille Jullian, 9, Presses de l'université de Provence, $186 \mathrm{p}$.

Columeau P., 1997a, « La faune archéologique du Port à Salses (66) dans le contexte de l'archéofaune du Languedoc occidental et du Roussillon au cours de l'âge du Fer », in Languedoc occidental protohistorique. Fouilles et recherches récentes ( $V I^{e}-I V^{e}$ s. av. J.-C.), Travaux du Centre Camille Jullian, 19, Presses de l'université de Provence, p. 11-22.

Columeau P., 1997b, « Mèze, Les Pénitents. Faune et modes d'approvisionnement en viande », in Languedoc occidental protohistorique. Fouilles et recherches récentes $\left(V I^{e}-I V^{e} s\right.$. av. J.-C.), Travaux du Centre Camille Jullian, 19, Presses de l'université de Provence, p. 151-156.
Columeau P., 2004, « La consommation de viande dans la ferme de Casse-Diables », in Ugolini D., Olive C., Béziers I (6oo-3oo av. J.-C.). La naissance de la ville, Catalogue d'exposition, 2006, Béziers, p. 80-81.

FOREST V., 2008, «Équidés de La Tène finale et de la période romaine en Gaule : approche ostéométrique », in BLANCHARD J.-L., IzAC-IMBert L. (DIR.), L'exploitation agricole dans son environnement à la fin de l'âge du Fer. Nouvelles approches méthodologiques, Actes de la table-ronde de SaintJulien, 18-19 novembre 2004, Toulouse, Archives d'écologie préhistorique, p. 61-71.

ForEST V., 2011, «Étude archéozoologique. Ostéologie, conchyliologie ", in RATSimba A. et al. (DIR.), Languedoc-Roussillon, Gard, Nîmes. Mas Vigier, Cadereau de saint Cézaire, Rapport final d'opération, Inrap/SRA Languedoc-Roussillon, t. $1 / 2$, p. 229-250.

FOREST V., 2012, « Étude archéozoologique : ostéologie, conchyliologie », in SÉJALON P. (DIR.), Languedoc, Gard, Nîmes, Mas de Vignoles IX, Rapport d'opération, Inrap/SRA Languedoc-Roussillon, t. I, vol. 1, p. 229-253.

GARDEISEN A., 1999, «Économie de production animale et exploitation du milieu à Lattes au cours du IV ${ }^{\mathrm{e}}$ siècle avant notre ère », in Py M. (DIR.), Recherches sur le quatrième siècle avant notre ère à Lattes, Lattara, 12, Lattes, p. 537-568.

Gardeisen A., 2002, « La faune », in Py M., Roure R., Le Cailar (Gard). Un nouveau comptoir lagunaire protohistorique au confluent du Rhôny et du Vistre, Documents d'Archéologie méridionale, 25, CNRS, p. 202-204.
GARDEISEN A., 2003, « Contribution de l'archéozoologie des grands mammifères à létude d'un espace ouvert en contexte urbain. La zone 123 (Lattes/SaintSauveur, Hérault) ", in Buxò R. et al., La place 123 de Lattara. Recherches pluridisciplinaires sur un espace urbain du IV siècle avant notre ère, Lattara, 16, Lattes, CNRS, p. 169-184.

GARDEISEN A., 2010, « Gestion des animaux de bouche au cours du $v^{e} s$. av. n. è. dans le Midi méditerranéen (475-375) : un aperçu lattois », in JANIN TH. (DIR.), Premières données sur le $V^{e}$ siècle avant notre ère dans la ville de Lattara, Lattara, 21, Lattes, CNRS, p. 419-428.

JANNORAY J., 1955, Ensérune: contribution à l'étude des civilisations préromaines de la Gaule méridionale, BEFAR, 181, Paris, de Boccard.

Labaune C., Magnin F., 2002, « Pastoral management vs. land abandonment in Mediterranean uplands : impact on land snails communities », Global Ecology \& Biogeography, 11, p. 237-245.

MARTIN S., 2011, « Résultats de l'analyse malacologique du site », in Ratsimba A. et al., op. cit., p. 251-263.

POMARÈDES H. et al., à paraître, « Le paysage périurbain à Nîmes (Gard, France) de la Protohistoire au Haut-Empire (vi ${ }^{\mathrm{e}}$ s. av. n. è.-II ${ }^{\mathrm{e}}$ s. de n. è.) ", in Plana R., BelaRTE C. (DIR.), Le paysage périurbain: définition et études de cas en Méditerranée et dans l'Europe continentale (monde grec, monde celte, monde romain), Actes du colloque international, Tarragone, 6-8 mai 2009.

Ratsimba A. et al., 2011, Mas Vigier, Cadereau SaintCézaire, Languedoc-Roussillon, Nîmes (3o), Rapport final d'opération, Inrap/SRA Languedoc-Roussillon, 2 vol., 334 et 382 p.

SÉJALON P. et al., 2009, « Définition et organisation des terroirs protohistoriques de Nîmes, Gard (de la fin du vi ${ }^{\mathrm{e}}$ au I ${ }^{\mathrm{er}}$ s. av. J.-C.) », in BERTRAND I. et al., Habitats et paysages ruraux en Gaule et regards sur d'autres régions du monde celtique, Actes du colloque AFEAF, Chauvigny, 17-20 mai 2007, t. II, Mémoire XXXV, APC, p. 153-18o.

SÉJAlon P. et al., 2012, Mas de Vignoles IX à Nîmes (Gard), Rapport final d'opération, Inrap/SRA Languedoc-Roussillon, 2 t., 4 vol. 